\title{
Infección por Mycobacterium tuberculosis en una niña sometida a trasplante de progenitores hematopoyéticos
}

Julia Palma, Paula Catalán, Patricia Mardones y M.Elena Santolaya

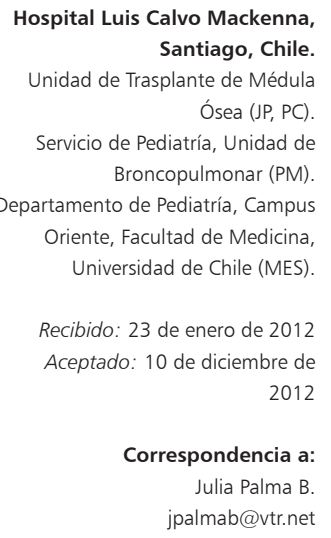

Julia Palma B. jpalmab@vtr.net

\section{Mycobacterium tuberculosis infection in a pediatric patient who underwent a hematopoietic stem cell transplant}

We report the case of a 10 year old girl with a relapsed acute lymphoblastic leukemia, who underwent a haploidentical hematopoietic stem cell transplant (HSCT), with grade II skin and digestive graft versus host disease, treated with corticosteroids and cyclosporine. On day +54 , she presented fever, with no other remarkable clinical findings. Imaging study showed the presence of lung and liver nodules, liver biopsy was performed. The study included histology, staining and culture for bacteria and fungi, and the preservation of a piece of tissue at $-20^{\circ} \mathrm{C}$ for future prospective studies. Ziehl Nielsen stain was positive, and study for Mycobacterium infection was performed. Microbiological smears of tracheal and gastric aspirate, and bronchial fluid obtained by bronchoalveolar lavage (BAL) were positive. The final report confirmed Mycobacterium tuberculosis in gastric content, sputum, BAL and liver tissue, susceptible to rifampin, isoniazid, streptomycin and ethambutol, with determination of mutations for genes rpo $\beta$ and kat $G(-)$. Tuberculosis (TB) diagnosis was confirmed. The girl received daily therapy for two months and then she continued on three times per week therapy for 9 months. Controlled by the transplant, infectious diseases and respiratory teams, the patient remained in good general condition, with radiologic resolution of pulmonary and liver involvement and negative smears. We conclude that Mycobacterium tuberculosis infection should be part of differential diagnosis of febrile illness in patients undergoing HSCT, and biopsy should be a standard practice of early diagnosis in these patients.

Key words: Mycobacterium tuberculosis, HSCT, liver nodules, biopsy.

Palabras clave: Mycobacterium tuberculosis, TPH, nódulos hepáticos, biopsia.

\section{Introducción}

$\mathrm{E}$ 1 trasplante de progenitores hematopoyéticos (TPH) es un procedimiento terapéutico validado en pediatría para patologías oncológicas y no oncológicas. La morbilidad y la mortalidad asociadas al TPH han disminuido en las últimas décadas debido al uso de regímenes de acondicionamiento menos tóxicos, cambios en las pautas de inmunosupresión y mejoría en los tratamientos de apoyo. Sin embargo, el riesgo de mortalidad asociado a infecciones y a la enfermedad injerto contra hospedero (EICH) aguda sigue siendo relevante ${ }^{1,2}$

En la actualidad, 80\% de los pacientes con leucemia linfoblástica aguda (LLA) cura con quimioterapia. Aquellos niños que no responden a la quimioterapia o que recaen son candidatos a TPH. Si un paciente se trasplanta de un hermano compatible, se obtiene curación a largo plazo en más de $50 \%$ de los casos ${ }^{3}$. La toxicidad acumulada con tratamientos de quimioterapia o la presencia de una alta carga tumoral reduce el número de pacientes susceptibles de someterse a un TPH convencional. Por otro lado, en países latinoamericanos dos tercios de los pacientes pediátricos no tienen un donante familiar compatible y no tienen acceso a donantes en los bancos internacionales de células hematopoyéticas, ya sea de médula ósea o de sangre de cordón umbilical, por constituir una minoría étnica con baja representación en los registros internacionales ${ }^{4}$. Para estos pacientes se han diseñado nuevas terapias, surgiendo el uso clínico del TPH familiar haploidéntico (el donante es el padre o madre que comparten un haplotipo con el receptor $)^{5,6}$.

\section{Caso clínico}

Paciente de 10 años, sexo femenino, con el antecedente de una LLA en segunda remisión completa, sometida a un TPH haploidéntico de sangre periférica depletada de linfocitos T.

En la Tabla 1 se presentan los resultados del estudio infectológico habitual en el período pre-trasplante de donante y receptor. La donante fue la madre de 41 años, sana, con serología (IgG) positiva para citomegalovirus (CMV) y virus de Epstein Barr (VEB), además de IgM positiva para toxoplasmosis, por lo que recibió terapia con espiramicina, con control negativo por reacción de polimerasa en cadena (RPC) previo a ser donante. Por su parte, la paciente tenía IgG positiva para CMV, VEB y virus herpes simplex tipo 1. No se efectuó estudio para tuberculosis. 
A su ingreso a la unidad de trasplante destacaba una niña eutrófica, en buenas condiciones generales, piel y mucosas sanas, con un catéter venoso central implantable con reservorio subcutáneo, examen cardiopulmonar, abdominal y neurológico normal, exámenes hematológicos normales; mielograma con $2 \%$ de blastos, función hepática, renal, ecocardiografia y espirometría normales. Recibió un acondicionamiento de intensidad reducida con fludarabina, thiotepa, melfalan, radioterapia nodal total, globulina anti-timocitos y rituximab.

Se realizó la infusión de progenitores hematopoyéticos depletados de linfocitos $\mathrm{T}$ sin incidentes. Como profilaxis de la EICH recibió ciclosporina y metilprednisolona en el primer período post TPH, como profilaxis antiinfecciosa recibió fluconazol $3 \mathrm{mg} / \mathrm{kg} /$ día, aciclovir $1.500 \mathrm{mg} /$ $\mathrm{m}^{2} /$ día, cotrimoxazol $20 \mathrm{mg} / \mathrm{kg}$ de sulfa, tres veces por semana desde el día +15 e inmunoglobulina iv, $400 \mathrm{mg} /$ kg semanal.

De acuerdo al protocolo de trasplante haploidéntico, se realizó seguimiento una vez por semana con galactomanano (GM) y carga viral para adenovirus (ADV), VEB y CMV desde el día de la infusión hasta el día +100 post TPH y luego bimensual hasta los 6 meses.

El día +4 presentó fiebre, calofríos, odinofagia y dolor en la cavidad oral. Al análisis de laboratorio destacaba un recuento absoluto de neutrófilos (RAN) de $0 / \mathrm{mm}^{3}$ y una proteína $\mathrm{C}$ reactiva $(\mathrm{PCR})$ de $149 \mathrm{mg} / \mathrm{L}$, por lo que se inició esquema antimicrobiano empírico con ceftazidima, amikacina y vancomicina. El hemocultivo central fue positivo para Staphylococcus coagulasa negativa. Completó 10 días de tratamiento estando la paciente afebril, en buenas condiciones generales, con RAN de $0 / \mathrm{mm}^{3}$, $\mathrm{PCR}<$ a $20 \mathrm{mg} / \mathrm{L}$, hemocultivos de control negativos y ecocardiograma normal.

Con respecto a la reconstitución hematopoyética, la paciente logró el implante con quimerismo $100 \%$ al día +7 , plaquetas $\left(>20.000 / \mathrm{mm}^{3}\right)$ y serie blanca $($ RAN $>500 /$ $\mathrm{mm}^{3}$ ) al día +10 y serie roja (reticulocitos $>1 \%$ ) al día + 11. El día +19 se diagnosticó una reactivación de CMV recibiendo tratamiento con ganciclovir iv por 21 días y luego en dosis de mantención hasta el día +50 , con cargas virales negativas.

Desde el día +41 presentó deposiciones líquidas y vómitos. Se planteó una probable EICH digestiva alta iniciándose tratamiento con prednisona. El estudio de deposiciones fue negativo para bacterias, rotavirus, ADV entéricos, Microsporidium y Cryptosporidium spp. Se realizó una endoscopia digestiva alta que mostró un edema leve y una imagen en empedrado en el antro gástrico. La biopsia confirmó una EICH digestiva de alta, grado I-II.

El día +54 , la paciente presentó nuevamente fiebre y compromiso de estado general, sin síntomas de localización al examen físico. Previa toma de cultivos, se inició terapia antimicrobiana empírica. Dentro de su estudio
Tabla 1. Estudio infectológico del donante y receptor pre trasplante de precursores hematopoyéticos

\begin{tabular}{lcc|} 
Examen & Donante & Receptor \\
Toxoplasmosis & - & - \\
Enfermedad de Chagas & - & - \\
Toxocariosis & - & - \\
VDRL & - & - \\
Hepatitis A & - & - \\
Hepatitis B & - & - \\
Hepatitis C & - & - \\
VIH & - & - \\
HTLV & - & - \\
CMV & $\operatorname{lgG}(+), \operatorname{lgM}(-)$ & $\operatorname{lgG}(+), \lg M(-), \operatorname{RPC}(-)$ \\
VEB & $\operatorname{lgG}(+), \lg M(-)$ & $\lg (+), \lg M(-), \operatorname{RPC}(-)$ \\
VHS 1 & $\operatorname{lgG}(-)$ & $\lg (-)$ \\
VHS 2 & $\lg (-)$ & - \\
ADV & - & \\
\hline
\end{tabular}

destacó una TAC de tórax con una lesión focal redondeada en la base pulmonar izquierda y dos adenopatías mediastínicas. En la TAC de abdomen el hígado presentaba múltiples lesiones focales hipodensas, redondeadas, con captación del medio de contraste en la periferia, distribuidas en forma aleatoria en el parénquima hepático, siendo la lesión de mayor tamaño de 1,3 cm de diámetro en el segmento VI (Figura 1).

En el contexto de un TPH haploidéntico con una EICH en tratamiento inmunosupresor activo, se realizó una biopsia hepática para estudio histológico, tinciones, cultivo bacteriano y fúngico, además de la conservación de un trozo de tejido a $-20^{\circ} \mathrm{C}$ para futuros eventuales estudios.

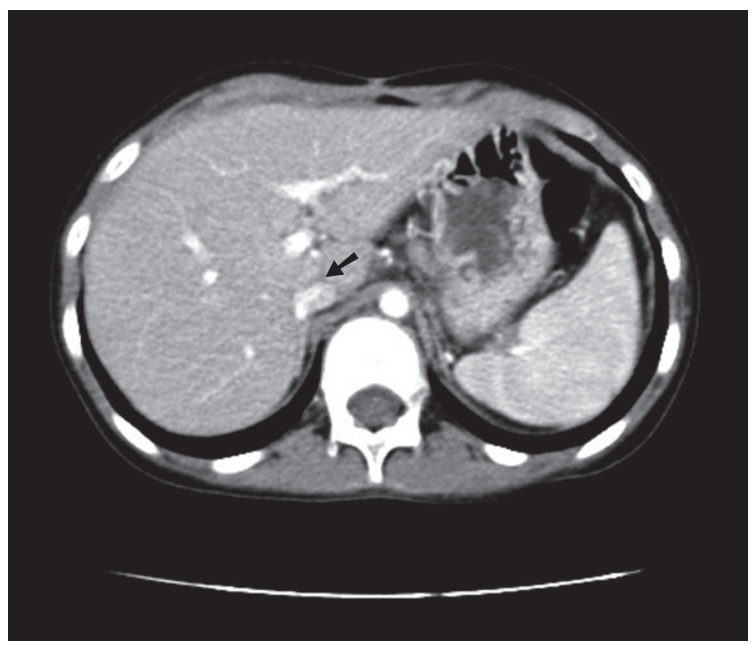

Figura 1. Tomografía axial computada de abdomen en paciente con cuadro febril en el día 54 post TPH haploidéntico (flecha). 
Figura 2. Baciloscopia positiva de aspirado traqueal. Se observan bacilos ácido alcohol resistentes (flechas).

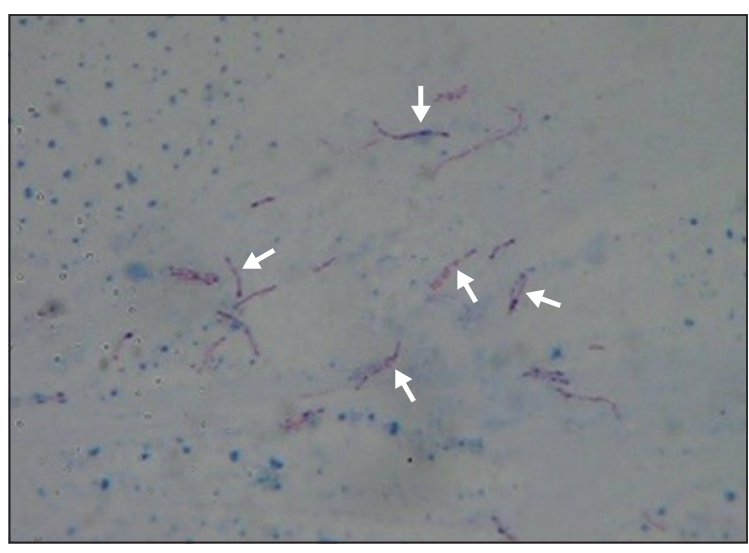

Se ajustó la cobertura antimicrobiana a meropenem, vancomicina y voriconazol. La paciente persistió febril, con compromiso del estado general, deposiciones disgregadas, sin síntomas respiratorios, con PCR en ascenso (170-194 $\mathrm{mg} / \mathrm{L}), \mathrm{GM}$ seriados negativos y ecocardiografía normal.

El estudio anátomo-patológico del tejido hepático mostró focos inflamatorios confluentes con formación de abscesos con infiltración linfohistiocitaria y polimorfonuclear, fibrina y necrosis. En torno a los focos inflamatorios, el parénquima hepático estaba disgregado, fibrinoso, sin células gigantes. Las tinciones de Gram y Grocott fueron negativas y la de Kinyoun resultó positiva.

En el estudio microbiológico, la tinción de Ziehl Nielsen fue positiva $(+++)$. El cultivo corriente fue negativo y se incubó la muestra de tejido para cultivo de Mycobacterium.

Se planteó el diagnóstico de una infiltración hepática nodular por Mycobacterium tuberculosis o Mycobacterium atípico. Se complementó el estudio con un fondo de ojo, baciloscopia de contenido gástrico y esputo y baciloscopia, cultivo y RPC para el complejo Mycobacterium de lavado broncoalveolar, además de hemocultivos para Mycobacterium.

De acuerdo a la hipótesis diagnóstica de infección por M. tuberculosis, se trasladó la paciente desde una sala con filtro de aire de alta eficiencia (HEPA) y presión positiva, a un aislamiento con filtro HEPA y presión negativa. Se suspendió la inmunosupresión y se inició tratamiento con isoniazida, rifampicina, pirazinamida y etambutol. En espera de la identificación microbiológica, y ante la duda de una infección por M. tuberculosis o Mycobacterium atípico, se asoció moxifloxacina y claritromicina.

Se realizó el estudio de contacto al grupo familiar de acuerdo a recomendación del equipo broncopulmonar, resultando negativo.

En el fondo de ojo se observó una lesión redondeada retinocoroídea bajo la mácula, no solevantada, amarillenta, bilateral. Las baciloscopias de contenido gástrico $\mathrm{y}$ aspirado traqueal fueron positivas $(+)$ y también del fluido bronquial obtenido por LBA $(+++)$ (Figura 2). La baciloscopia de orina y LCR fueron negativas. El cultivo enviado al Instituto de Salud Pública de Chile confirmó el crecimiento de $M$. tuberculosis en contenido gástrico, esputo, LBA y tejido hepático, susceptible a rifampicina, isoniazida, estreptomicina y etambutol con determinación de mutaciones de genes rpo $\beta$ y kat $G(-)$. Los hemocultivos para Mycobacterium spp. resultaron negativos.

Ante esta confirmación microbiológica, se ajustó el tratamiento a cuatro fármacos (isoniazida, rifampicina, pirazinamida y etambutol). Al completar un mes de tratamiento, la paciente se encontraba afebril, en buenas condiciones generales y con buena tolerancia a la terapia. Las baciloscopias de control fueron negativas, la TAC de cerebro, senos paranasales, tórax y pelvis no mostraron lesiones, y la TAC de abdomen presentaba una regresión significativa de las lesiones nodulares hepáticas. Completó dos meses de tratamiento diario (hasta el día +112 post $\mathrm{TPH}$ ), luego de lo cual se ajustó la terapia a la modalidad trisemanal con isoniazida, rifampicina y etambutol. Controlada por el equipo de trasplante, infectología y broncopulmonar, la paciente evolucionó en buenas condiciones generales, con imágenes que mostraron resolución del compromiso hepático y pulmonar, manteniendo baciloscopias negativas. Completó 11 meses de tratamiento antimicrobiano. En su evaluación a dos años post-trasplante, la paciente estaba inmunoreconstituida, sin evidencias de reactivación de la infección tuberculosa.

\section{Discusión}

Este caso representa la realidad del TPH haploidéntico, una herramienta terapéutica eficiente para pacientes pediátricos en Chile, con un alto riesgo de morbimortalidad relacionado a enfermedades infecciosas, considerando el uso de fármacos inmunosupresores como parte del acondicionamiento y del post TPH y la lenta inmunoreconstitución a la que se ven expuestos estos pacientes ${ }^{7,8}$.

En este contexto, la infección por M. tuberculosis debe formar parte del diagnóstico diferencial de los cuadros febriles en los pacientes sometidos a $\mathrm{TPH}^{9,10}$. El hallazgo de una tuberculosis en esta paciente plantea algunas interrogantes y reflexiones epidemiológicas, diagnósticas y terapéuticas.

Desde el punto de vista epidemiológico, se asume que la tuberculosis en el niño es siempre reflejo de una infección reciente y este concepto obliga a la búsqueda de un posible caso infectante entre los contactos del menor ${ }^{11}$. En el caso de nuestra paciente, se realizó el estudio de todos sus contactos intrafamiliares, el que fue negativo. Una segunda opción, por tratarse de una paciente intensamente inmunocomprometida, es que se trate de una reactivación de una posible primoinfección previa, lo que parece más probable en el caso de esta niña. 
En la esfera del diagnóstico, es relevante enfatizar que la toma de biopsia con estudio microbiológico debe ser una práctica habitual y precoz en el enfrentamiento diagnóstico de pacientes inmunocomprometidos que presentan lesiones nodulares en algún parénquima asequible a ser biopsiado $^{12}$.

Con respecto al tratamiento, el esquema terapéutico primario recomendado por la OMS en el año 2010 contempla una fase intensiva (dos meses) y una de continuación (cuatro meses). La primera fase requiere terapia diaria con cuatro fármacos antituberculosos (isoniazida, rifampicina, pirazinamida y etambutol) y la fase de continuación recomienda esquemas variables, con dosis diarias de isoniazida-rifampicina, o con terapia trisemanal con tres o cuatro medicamentos ${ }^{13}$. En el caso de terapia antituberculosa a pacientes con deficiencias del sistema inmune, se recomienda prolongar el tratamiento completando al menos nueve meses, con el propósito de disminuir la tasa de recaídas ${ }^{14}$. En el caso de nuestra paciente se decidió prolongar la fase de continuación con tres fármacos en esquema trisemanal hasta completar 11 meses totales de tratamiento, basado en la opinión de expertos, considerando la persistencia de su compromiso inmunológico, su lenta inmunoreconstitución y la persistencia de su EICH.

Dentro del estudio pre-trasplante, detallado anteriormente, a la fecha no se incluye dentro de la evaluación el status tuberculoso de estos pacientes. A la luz de este caso, de la población blanco y de los cambios epidemiológicos, nos parece importante revisar la evaluación de tuberculosis latente y redefinir de acuerdo a la evidencia existente el estudio de los candidatos a trasplante de progenitores hematopoyéticos

\section{Resumen}

Se presenta el caso clínico de una niña de 10 años, con una leucemia linfoblástica aguda en recaída, sometida a un trasplante de progenitores hematopoyéticos (TPH) haploidéntico, con enfermedad injerto contra hospedero cutánea y digestiva grado II, en tratamiento con corticosteroides y ciclosporina, que presentó el día +54 posttrasplante fiebre y compromiso de estado general. Dentro del estudio de su cuadro febril se practicaron imágenes que mostraron presencia de nódulos pulmonares y hepáticos. Se realizó una biopsia hepática cuyo estudio incluyó histología, tinciones y cultivo para bacterias y hongos. La tinción de Ziehl Nielsen de tejido hepático, así como las baciloscopias de contenido gástrico, aspirado traqueal y de fluido bronquial obtenido por lavado broncoalveolar (LBA) fueron positivas. El informe definitivo de cultivo confirmó Mycobacterium tuberculosis en contenido gástrico, esputo, LBA y tejido hepático, sensible a rifampicina, isoniazida, estreptomicina y etambutol, con determinación de mutaciones de genes rpo $\beta$ y kat $G(-)$. Se confirmó el diagnóstico de tuberculosis, por lo que recibió tratamiento diario con cuatro fármacos por dos meses y luego terapia trisemanal con rifampicina, isoniazida y etambutol por nueve meses. Controlada por los equipos de trasplante, infectología y broncopulmonar, la paciente se mantiene actualmente en buenas condiciones generales, con imágenes con resolución del compromiso hepático y pulmonar y baciloscopias negativas. La infección por $M$. tuberculosis debe formar parte del diagnóstico diferencial de los cuadros febriles en los pacientes sometidos a TPH, y la toma de biopsia debe ser una práctica habitual y precoz en el enfrentamiento diagnóstico de estos pacientes.

\section{Referencias bibliográficas}

1.- Styczynski J, Gil L. Prevention of infectious complications in pediatric hematopoietic stem cell transplantation. EBMT Pediatric diseases Working party. Bone Marrow Transplant 2008; 42 Suppl 2: S77-81.

2.- Verneris M R, Eapen M, Duerst R, Carpenter P A, Burke M J, Afanasyev B V, et al. Reduced-intensity conditioning regimens for allogeneic transplantation in children with acute lymphoblastic leukemia. Biol Blood Marrow Transplant 2010; 16: 1237-44.

3.- Hough R, Rocha V. Transplant outcomes in acute leukemia. II. Semin Hematol 2010; 47: 51-8.

4.- Rocha V, Locatelli F. Searching for alternative hematopoietic stem cell donors for pediatric patients. Bone Marrow Transplant 2008; 41: 207-14.

5.- Smith A R, Baker K S, Defor T E, Verneris M R, Wagner J E, Macmillan M L. Hematopoietic stem cell transplantation for children with acute lymphoblastic leukemia in second complete remission: similar outcomes in recipients of unrelated marrow and umbilical cord blood versus marrow from HLA matched sibling donors. Biol Blood Marrow Transplant 2009; 15: 1086-93.

6.- $\quad$ Leung W, Campana D, Yang Y, Pei D, Coustan-Smith E, Gan K, et al. High success rate of HSCT regardless of donor source in children with very high-risk leukemia. Blood 2011; 118: 223-30.

7.- Barrett J, Gluckman E, Handgretinger R, Madrigal A. Point-cointerpoint: haploidentical family donors versus cord blood transplantation. Biol Blood Marrow Transplant 2011; 17 (1 Suppl): S89-93.

8.- Cairo M, Rocha V, Gluckman E, Hale G, Wagner J. Alternative allogeneic donor sources for transplantation for childhood diseases: unrelated cord blood and haploidentical family donors. Biol Blood Marrow Transplant 2008; 14 (Suppl 1): 44-53.

9.- González B, King A, Dal Borgo P. Trasplante de médula ósea en pacientes portadores de inmunodeficiencias combinadas. Rev Chil Ped 2000; 71: 32-40.

10.- Golden M P, Vikram H R. Extrapulmonary tuberculosis: an overview. Am Fam Physician 2005; 72: 1761-68

11.- Swaminathan S, Rekha B. Pediatric tuberculosis: global overview and challenges. Clin Infect Dis 2010; 50 (Suppl 3): S184-94.

12.- Muñoz A, González-Vicent M, Badell I, Díaz de Heredia C, Martínez A, Maldonado M. Mycobacterial diseases in pediatric hematopoietic SCT recipients. Bone Marrow Transplant 2011; 46: 766-8.

13.- World Heath Organization. Treatment of tuberculosis. Guidelines, 4th ed. Geneva, Switzerland 2009. WHO/HTM/ TB/2009.420.

14.- Panjabi R, Comstock G W, Golub J E. Recurrent tuberculosis and its risk factors: adequately treated patients are still at high risk. Int J Tuberc Lung Dis 2007; 11: 828-37. 